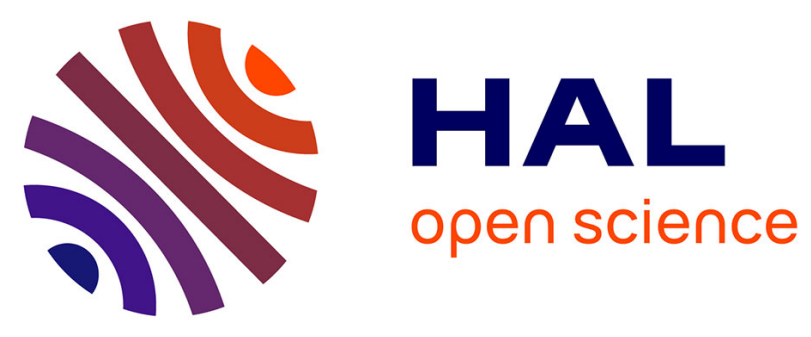

\title{
In Touch with Everyday Objects: Teleportation Techniques in Virtual Environments Supporting Tangibility
}

Yiran Zhang, Sy-Thanh Ho, Nicolas Ladeveze, Huyen Nguyen, Cédric Fleury, Patrick Bourdot

\section{To cite this version:}

Yiran Zhang, Sy-Thanh Ho, Nicolas Ladeveze, Huyen Nguyen, Cédric Fleury, et al.. In Touch with Everyday Objects: Teleportation Techniques in Virtual Environments Supporting Tangibility. Workshop on Everyday Virtual Reality (WEVR), IEEE Conference on Virtual Reality and 3D User Interfaces, Mar 2021, Lisbon (virtual), Portugal. pp.278-283, 10.1109/VRW52623.2021.00057 . hal-03328157

\section{HAL Id: hal-03328157 https://hal.science/hal-03328157}

Submitted on 28 Aug 2021

HAL is a multi-disciplinary open access archive for the deposit and dissemination of scientific research documents, whether they are published or not. The documents may come from teaching and research institutions in France or abroad, or from public or private research centers.
L'archive ouverte pluridisciplinaire HAL, est destinée au dépôt et à la diffusion de documents scientifiques de niveau recherche, publiés ou non, émanant des établissements d'enseignement et de recherche français ou étrangers, des laboratoires publics ou privés. 


\section{In Touch with Everyday Objects: Teleportation Techniques in Virtual Environments Supporting Tangibility}

\author{
Yiran Zhang * \\ University Paris-Saclay, \\ CNRS, LISN, \\ VENISE team, Orsay, France
}

\author{
Sy-Thanh Ho \\ University Paris-Saclay, \\ CNRS, LISN, \\ VENISE team, Orsay, France
}

\author{
Cédric Fleury \\ University Paris-Saclay, \\ CNRS, Inria, \\ LISN, Orsay, France
}

\author{
Nicolas Ladévèze \\ University Paris-Saclay, \\ CNRS, LISN, \\ VENISE team, Orsay, France \\ Patrick Bourdot \\ University Paris-Saclay, \\ CNRS, LISN, \\ VENISE team, Orsay, France
}

\author{
Huyen Nguyen \\ University Paris-Saclay, \\ CNRS, LISN, \\ VENISE team, Orsay, France
}

\begin{abstract}
The application of virtual reality (VR) for everyday use is often limited due to the lack of tactile and kinesthetic feedback. To facilitate and expand the use of VR in daily life, it is possible to employ physical objects readily available at home as tangible objects to provide this missing feedback. For instance, a real chair can allow a user to sit within the virtual environment, even if the sitting place in the virtual world is not a chair. In home-based games, a real but not dangerous stick can provide the holding sensation of a virtual sword. These tracked objects in the real world can serve as a tangibility medium to their virtual counterparts, contributing to a higher sense of presence and immersion. However, such a solution relies on a consistent spatial relationship between the real and virtual space surrounding the user, which makes the basic use of virtual navigation techniques such as teleportation difficult. To allow the navigation on a large virtual environment while supporting a tangible interaction with real objects in a limited physical space at home, this paper explores three different teleportation techniques: to teleport the user, the object, or both of them to a new position accordingly while preserving the user and object's spatial relationship. All of these approaches enable tangible interaction when using a teleportation technique for navigation, but each one is more or less suitable in different situations of real/virtual spatial consistency.
\end{abstract}

Index Terms: Human-centered computing-Human computer interaction (HCI) - Interaction paradigms - Virtual reality; Human-centered computing-Human computer interaction (HCI)Interaction techniques

\section{INTRODUCTION}

The use of virtual reality (VR) has been steadily grown in our daily life, such as at homes and offices, thanks to the affordability of off-the-shelf display headsets and the exponential development in applications and games. This growth, therefore, emphasizes the urgency to consider and study user experience for their adoption of VR applications in everyday life. Haptic technology is one of the VR technologies whose objective is to enhance the realism and immersion of user experience with a sense of touch [11].

Indeed, many haptic devices on the market use different modalities and techniques to provide a user with tactile sensation, including

*This is the authors' version of the work. It is posted here for your personal use. Not for redistribution. The definitive version of record was published in 2021 IEEE Conference on Virtual Reality and 3D User Interfaces Abstracts and Workshops (VRW), 2021, pp. 278-283, doi: 10.1109/VRW52623.2021.00057. pressure, skin stretch, vibration, and temperature. As opposed to dedicated VR setups found in academic labs or big companies, these devices are not always available at home or in SME offices, and they are quite expensive for the general public. Therefore, tactile and kinesthetic feedback is usually absent in home-based VR applications.

To address this limitation, some previous works propose to integrate everyday objects into a virtual environment by pairing them with their virtual counterparts. For example, an umbrella can represent Darth Vader's lightsaber [20], or a suspended ball can be deployed as a flying droid that physically attacks the user [7]. The main idea is to employ some tangible objects in the real working space, which provide passive haptic feedback [11] realistic enough in terms of tactile-kinesthetic sensation. Therefore, the user can perceive a haptic feeling when touching the virtual objects. This simple and cost-effective approach enhances the perception of virtual object affordances [9], improves the user's sense of presence, and contributes to a more compelling VR experience. However, this approach is also subjected to its limitations when applied in some scenarios, particularly accompanied by virtual-navigation techniques $[4,6,10,22]$.

In our context, enabling the user to touch a virtual object via a tangible prop highly relies on the consistent spatial relationship between the user, the tangible object, and its virtual counterpart. Although allowing the user to carry out virtual navigation is mandatory in many large-scale virtual environments, a major issue is that such techniques easily break the one-to-one mapping between the virtual and real space surrounding the user. Consequently, after the virtual navigation, the tangible objects will no longer be located at their counterpart position in the virtual world. Once the user releases a tangible object before the virtual navigation, it becomes impossible for them to find and touch it again afterwards while still wearing the VR headset. Therefore, virtual navigation techniques are usually excluded when there are tangible objects involved, limiting the user's available interaction space in the virtual environment to their real space.

In this paper we propose three interactive techniques to allow the user to navigate in a large-scale virtual environment while preserving the possibility to interact with virtual objects and their real/tangible counterparts. These techniques are to some extent based on the basic teleportation navigation technique [4], this latter being widely used in many VR applications and known as limiting simulator sickness compared to other virtual navigation techniques [10,22]. Our techniques aim to teleport either the user, the virtual object, or both of them to a new position, to recover the spatial relationship which makes a consistent mapping between the user, the virtual object, and its tangible counterpart possible after teleportation. 


\section{Related Work}

Passive haptics [11] allows the user to have tactile and kinesthetic feedback in the virtual world by physically touching a tangible object in the real world. This concept has been widely used in many VR applications. For example, the iTurk system [7] complements the VR experience with a foldable object as a physical prop that can be reconfigured as a suitcase or a fuse box in the virtual world. This iTurk system focuses on the mapping of only one physical object to several virtual objects of the similar form and shape. Meanwhile, the Substitutional reality system [20] pairs every physical object and architectural feature surrounding the user to its virtual counterpart, but still allows some degree of discrepancy between them.

Adding physical qualities to virtual objects can enhance the sense of presence of the user. Indeed, in the study of Hoffman et al. [11], participants who were allowed to "touch and see" the object had more accurate prediction of object properties than those who were only allowed to "see" it. Insko et al. [12] showed that augmenting a visual-cliff environment with a real wooden plank as a ledge significantly impacted participants' behavioral presence, heart rate, and skin conductivity compared to a virtual-ledge-only condition. Moreover, in their experiment, they demonstrated that using passive haptics could lead to significant increase in presence and spatial knowledge training transfer. Besides, passive haptics can also be applied in medical fields. For example, allowing patients to touch a spider replica's furry texture can enhance the treatment of spider phobia [5]. In the work of Kotranza and Lok [17], augmenting a virtual patient with a tangible interface makes the interaction during a breast exam more similar to human-human interaction.

Passive haptics usually requires a one-to-one mapping between real and virtual object. This mapping limits the use of passive haptics in many VR applications that may introduce spatial discrepancies between the user's real and virtual world, for instance, when using virtual navigation in large-scale virtual environments. Moreover, a physical prop can often match only one specific type of virtual objects, limiting its use in a complex VR experience. These problems can be overcome by reconfiguring the physical prop during its use with the help of robots or human actuators. For example, robotic graphics [19] uses a robotic arm to correctly place a real object in front of the user's hand when they try to reach for a virtual object. Snake charmer [1] not only aligns a robotic arm with the object's virtual representation but also allows the user to feel one or more of object's shape, texture, and temperature. In addition to using robots, some applications such as TurkDeck [8] employ a group of human workers to operate props to create a physical world adapting to the user's activities in the virtual world. However, using robot technologies or employing a team of human actuators are difficult in everyday VR experience.

Some redirected techniques can solve the passive haptic inflexibility. For example, redirected touching $[15,16]$ and haptic retargeting [3] introduce a discrepancy between the user's real and virtual hand motion. As the user's hand movement in the virtual environment is no longer the same as their real-world motion, the real and virtual hands can reach the real and virtual objects at the same time. By generating different mappings between the real and virtual spaces, a single physical object can provide haptic feedback for various virtual objects with different shapes. However, people also reported strange sensations as the virtual space is warped to map a variety of virtual objects onto a single real object, and a phase of adaption is necessary to achieve the same task performance as in the non-warped condition [16].

In the context of navigation by real walking, redirected walking [14] enables a single real prop to provide tangibility to multiple virtual objects by introducing an offset between the user's physical and virtual path. Although the user is usually not aware of the offset, this technique often requires physical spaces larger than $6 \mathrm{~m} \times 6 \mathrm{~m}[2]$, and is therefore not suitable for domestic use. For a compact tracking volume, Impossible space [21] deploys a selfoverlapping architectural layout of the virtual environment to allow the user to walk through multiple rooms via portals. The adjacent virtual room is physically overlapping with the previous one, allowing the user to reuse the same physical props in the new room. However, the specific architectural layout also imposes limitations on the design of the virtual environment.

Based on the limitations observed in these previous research works regarding everyday and/or domestic uses, this paper proposes three interaction techniques and discusses their complementary interests to help the user recover the spatial consistency during teleportation, with the tangible interaction support. Our techniques are suitable for small tracking volumes, such as domestic rooms and offices, and do not depend on a specific virtual environment layout. In addition, a single physical object can be reused to link with multiple similar virtual objects located in different places without introducing an offset.

\section{TANGIBILITY SUPPORTED BY TELEPORTATION}

The basic idea of our techniques is to teleport either the user, the virtual object, or both of them at once to a new position calculated based on the spatial relationship between the tangible object and the user. Indeed, the relationship between the user and the virtual object must be aligned with the spatial arrangement between the user and the tangible prop to recover the consistency and coherence of these components after the teleportation. Moreover, when tangible interactions are not required, during basic virtual navigation for example, the surrounding real objects are not aligned with any visual ones. Therefore, by using the spatial consistency recovering technique, the potential collisions between the user and those real-world tracked objects can be avoided using some specific visual feedback [18].

In the following sub-sections, we will present the design and implementation of three different advanced teleportation techniques to support tangible interaction. As an example of possible applications of these teleportation techniques, we designed a VR environment that incorporates a tracked physical chair in the real world to allow the user to rest on it. This chair also has a virtual counterpart with which the user can interact in the virtual world. However, the use cases associated with each technique can be extended into more general situations in everyday VR applications with different physical objects involved.

The VR setup of our proof-of-concept implementation consisted of an HTC Vive headset and two controllers. The virtual environment was rendered using Unity 2019.4.2f1 with a resolution of $1080 \times$ 1200 pixels per eye at $90 \mathrm{~Hz}$. The room supported a $3 \mathrm{~m} \times 5 \mathrm{~m}$ tracking area, and the real chair was tracked using one Vive Tracker.

Depending on the working context in the virtual environment, the user can select from a virtual menu at runtime the teleportation technique they want to use to connect virtual objects with their tangible counterparts. For instance, to interact with the virtual object and its physical counterpart in its current virtual context, the user can teleport himself close to the targeted object. On the contrary, if the current interaction context is not important to the user and the task at hand, the second technique can be applied to bring the object close to the user. Finally, if the user wants to use a virtual object at another place in the virtual environment, the hybrid technique can be activated.

\subsection{Teleportation of the User}

In this technique, the selected virtual object becomes an anchor of the teleportation. The system will calculate the user's avatar future position to reflect their actual position related to the physical object in the real world (see Fig. 1). After teleportation, the user can reach and interact with the real object using its virtual counterpart thanks to the recovered spatial consistency. 


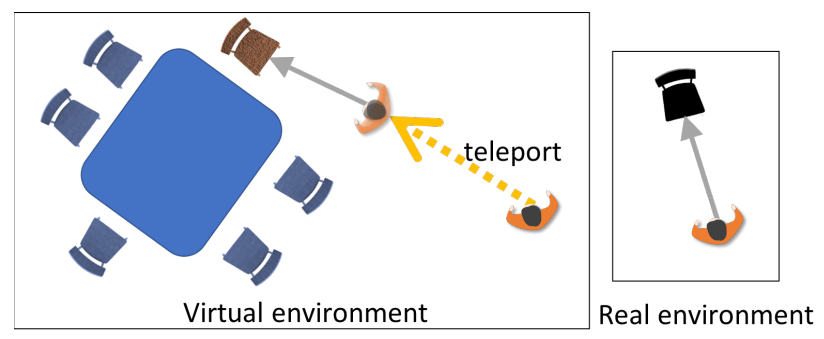

Figure 1: Teleportation of the user's position in the virtual world towards the targeted virtual object's (brown chair) position illustrated from the bird-eye view. Their new position recovers the spatial relationship between them and the object's physical counterpart (black chair).

To preserve the existing spatial relationship in the real world, the number of the possible future positions for the user's avatar in the virtual environment is decided by the number of the physical props integrated as well as their forms, shapes and potential mobility. For example, if we pair the ball suspended from a long pendulum with a virtual flying droid as in iTurk system [7], the number of possibilities to position the user near the ball in the virtual environment tends to be infinite. However, in Substitutional reality system [20], the mapping is unique since every architectural feature should pair to its virtual object. Besides, the possible future positions of the user's avatar also depend on the actual context of the virtual scene if the system wants to maintain a semantic coherence. For example, the system can help prevent the user from being positioned behind a virtual obstacle or in the middle of surrounding virtual object after the teleportation.

Although the user has the possibility to select an appropriate position amongst multiple options computed by the system, it is sometimes unavoidable when the user has no choice but to teleport into a virtual object to maintain the spatial relationship. In particular, it often happens when many virtual objects surround the target one. However, teleporting the user inside or in the middle of a virtual object can break the user's immersion and affect the realism of the virtual environment and VR experience. In order to overcome this issue, the system can show the user's future positions while highlighting the objects currently overlapping with them. The system then guides the user to physically move to a more appropriate position in the real world before triggering the teleportation.

In our implementation, the virtual object with potential tangibility (i.e., the chair) will be highlighted with a different color when the user hovers the laser pointer over it (see Fig. 2). The user can select the virtual object with the pointer and soft trigger the teleportation by pressing the "Grab" button on the Vive controller. Due to the shape of the chair (with backrest), the user has only one possible mapping position for the teleportation. If the user's future position of teleportation overlaps with the nearby virtual objects (e.g., the table), the user needs to physically move (i.e., walk) to a more appropriate position in the real world to be able to complete the teleportation. To achieve this, the system will display a clone of the distant context (the chair and the table that currently overlaps with user's future position) around the user's current position to simulate the scene after teleportation. The available area where the user can physically walk turns green, guiding the user to move to a position to avoid any overlapping. Once the user physically reach a position which is free of obstacles, the teleportation can be hard triggered by pressing the "Squeeze" button and the user will be teleported nearby the targeted tangible object while avoiding teleporting into the surrounding obstacles.

\subsection{Teleportation of the Object}

In this technique of teleportation, instead of moving the user towards the virtual object as mentioned in Section 3.1, it is also possible to
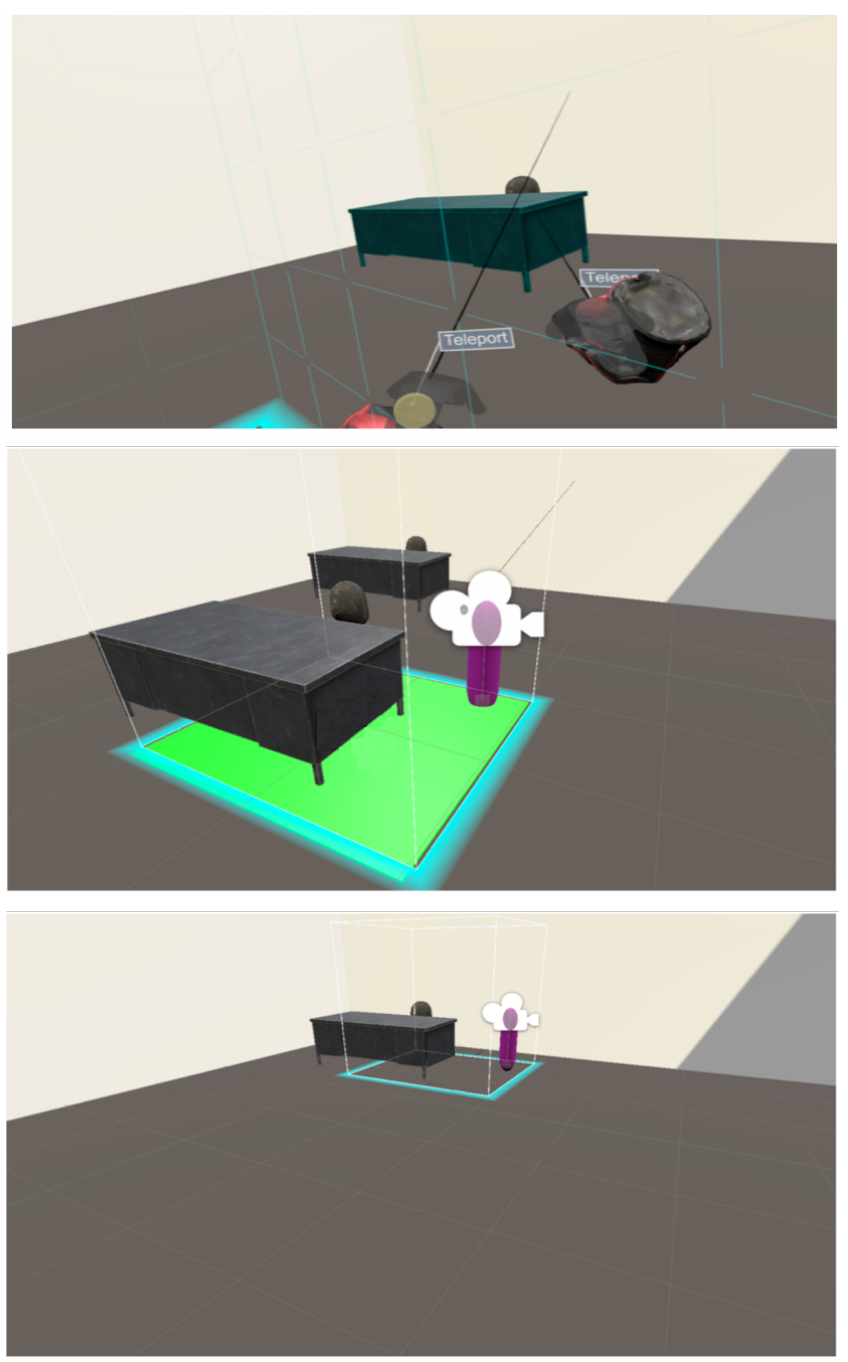

Figure 2: Top: the user selects the targeted object (brown chair) to soft trigger the teleportation. Middle: clones of the targeted chair and the surrounding table are displayed close to the user (purple avatar) to inform them of the situation after teleportation. The available walking area turns green to guide the user to a more suitable position to avoid overlapping. Bottom: after physically positioning themself in an appropriate position, the user hard triggers the teleportation and is teleported to the next position while maintaining a consistent spatial relationship with the tangible object.

bring the targeted virtual object to the user to recover the spatial relationship relating to the tangible object. Based on the actual position of the user's avatar in the virtual environment, we can compute the virtual object's future position in relation to its tangible prop (see Fig. 3). Different from the first technique which teleports the user's avatar, the resulted position is unique in this case. However, this position can be probably occupied or blocked by other virtual obstacles (e.g., a virtual wall) surrounding the user. In this case, the system detects post-teleportation interference and computes a new position to which the user has to teleport themself before completing the object-based teleportation operation.

To compute such position, the system first finds a new position for the targeted virtual object outside the obstacle. A basic approach is to use the point on the obstacle's collider that is closest to the virtual object's current teleportation destination. Then, based on this new position of the targeted virtual object, the system computes the new corresponding position for the user's avatar in relation to their 


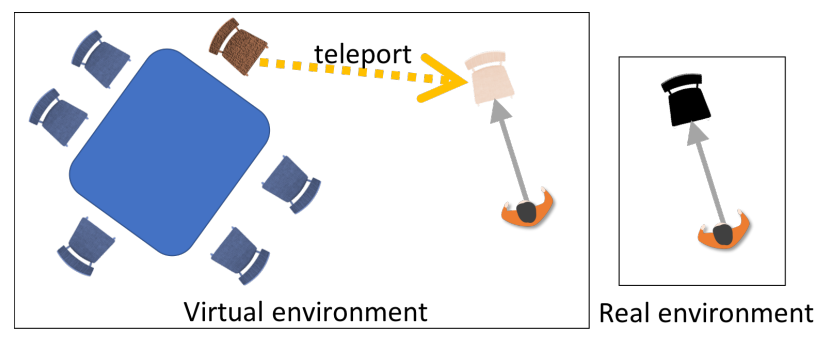

Figure 3: Teleportation of the targeted virtual object (brown chair) towards to the user's actual position illustrated from the bird-eye view. Its future position recovers the spatial relationship between the user and the object's physical counterpart (black chair).

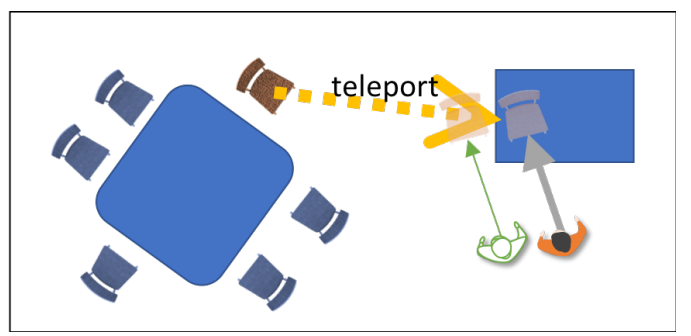

Figure 4: Basic approach to compute a valid teleportation destination for the user in the case of the object appearing in a virtual obstacle using Collider.ClosestPoint function of Unity3D.

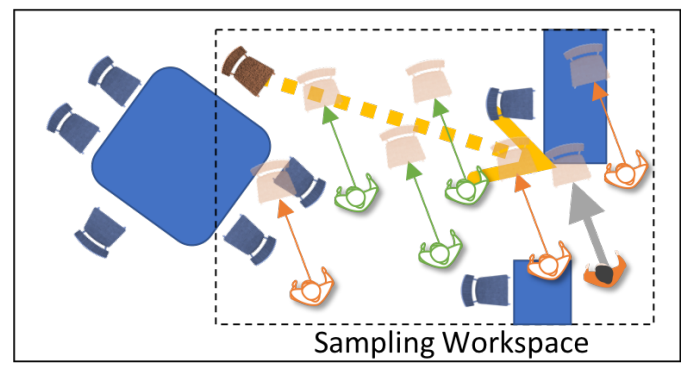

Figure 5: Computation of possible teleportation destinations (in green) using the PRM-based sampling approach on the couple of the virtual chair and the avatar of the user.

physical position in the real world (see Fig. 4). Consequently, the targeted virtual object will no longer overlap or be blocked by other virtual objects after the teleportation. However, in some crowded virtual environments, the resulted user's position computed from this basic approach can also be occupied by other obstacles in the scene (see Fig. 5). In this case, another configuration for the couple of virtual chair and the avatar of the user can be computed using the Probabilistic RoadMap (PRM) sampling approach [13].

Similar to the first technique, our implementation allows the user to select the targeted virtual object (i.e., the chair) and teleport the object towards the user. By pressing the "Grab" button on the controller, the user can soft trigger the teleportation. The system will show a clone of the targeted object at the computed position to inform the user of its future position (see Fig. 6). If the virtual object destination is occupied or blocked by other virtual objects, those obstacles will be highlighted. In this case, the user is then asked to teleport himself to a new position (presented as a green zone on the ground) in the virtual world before teleporting the object. After reaching such suitable virtual position, the user can hard trigger the
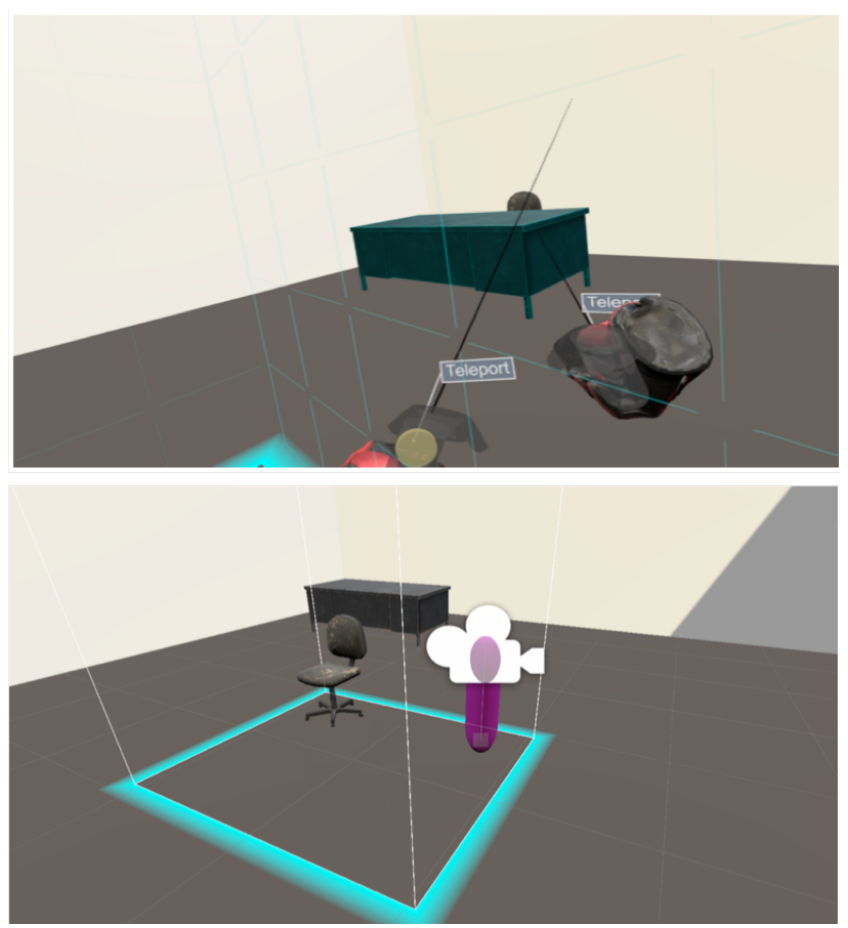

Figure 6: Top: the user selects the targeted virtual object (brown chair) to soft triggers the teleportation of the object. Bottom: a clone of the virtual object is shown in the surrounding of the user (purple avatar). The targeted virtual object is teleported toward to user after the user hard triggers the teleportation.

teleportation of the object with the "Squeeze" button. The targeted virtual object then will be teleported nearby the user.

\subsection{Hybrid Teleportation Technique}

As the name suggests, the hybrid technique is an ultimate approach to resolve the spatial consistency recovery problem by combining the first two techniques mentioned in Section 3.1 and 3.2. Similar to group teleportation [23], this technique allows the user to teleport the targeted virtual object and themself simultaneously to support a tangible interaction at a specific destination in the virtual world. However, their spatial relationship needs to be recomputed with regard to the spatial arrangement between the user and the physical prop (see Fig. 7).

Our hybrid technology deploys a virtual representation that couples the teleportation destinations of the user and the virtual object according to the current relative position between the user and the tangible object. By manipulating such virtual representation's position and rotation, the user can simultaneously define the teleportation destination for the targeted virtual object and themself. Thus, the user can customize the teleportation for different virtual contexts, optimizing the obstacle-related situations (e.g., obstruction, overlapping). At the same time, the teleportation provides tangible interactions for the user.

Unlike the other two implementations, the user soft triggers the teleportation with the "Pinch" button after selecting the targeted virtual object (i.e., the chair). The user can define their future position using the laser pointer. Based on this position, the system will calculate the corresponding position of the virtual object to maintain the spatial relationship between the physical object and the user, and display a clone of the target virtual object at the resulting position (see Fig. 8). By pressing the "Touch left" and "Touch right" buttons of the other controller, the user can rotate the clone object around the 


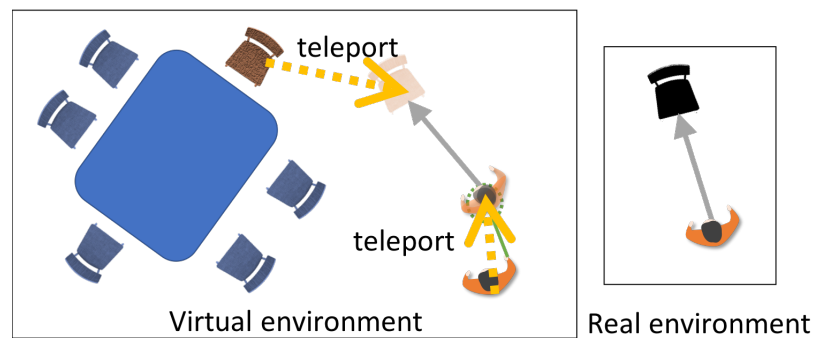

Figure 7: Hybrid teleportation of both the user and the targeted virtual object (brown chair) to their new positions defined by the user while preserving spatial relationship between them.
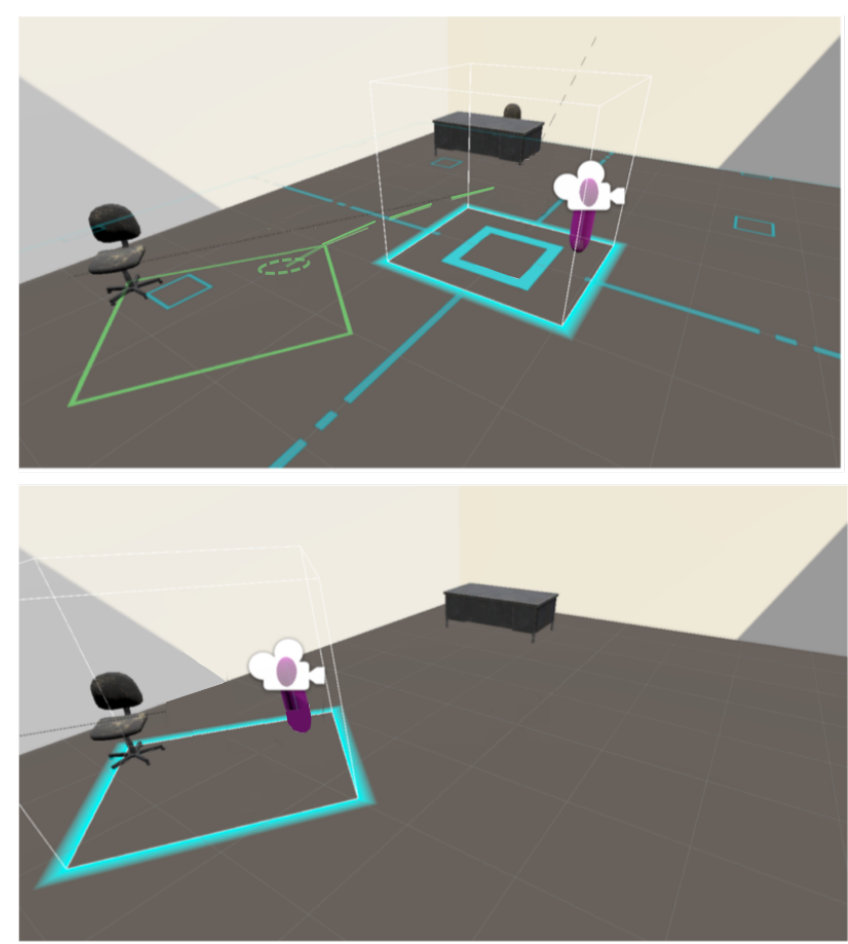

Figure 8: Top: when the user soft triggers the technique, the system displays a clone object at a location calculated based on the user-defined teleportation position. The user has all the freedom to manipulate the future position and orientation of the user and object as a set. Bottom: when the user hard triggers the technique, both the user and the object arrive at the predefined positions with a recovered spatial relationship.

their future position to make sure that the user or the object will not teleport into any other obstacles afterwards. Finally, by pressing the "Pinch" button again, the user and the virtual object can be teleported to the predefined position with a recovered spatial relationship.

\section{CONCLUSION}

Allowing a user to touch or hold virtual objects can enhance immersion and contributes to a greater sense of presence. Passive haptic is a simple and cost-effective solution to compensate the lack of haptic feedback in domestic use of virtual reality by using everyday objects as tangible props for virtual objects. However, passive haptic requires a one-to-one mapping between tangible props and their virtual counterparts, which usually hinders virtual navigation in large-scale virtual environments.
To overcome this issue, we propose three techniques to help the user to recover the spatial consistency between a targeted virtual object and its physical counterparts after teleportation: teleportation of the user, teleportation of the object and hybrid teleportation. Decided by the targeted virtual object's position, teleportation of the user technique teleports the user to a position near the virtual object to restore the spatial relationship. Different positions can be proposed to the user according to the shape, size and number of integrated tangible props. Based on the user's position in the virtual environment, teleportation of the object technique computes the virtual object's position corresponding to its tangible prop, and teleports the object to this unique position. Finally, hybrid teleportation approach combines these two techniques and allows the user to define a new teleportation destination for both the object and themself while restoring the spatial consistency.

These different techniques can be applied in different scenarios according to the targeted object's features. For example, teleportation of the user can be more appropriate than teleportation of the object or hybrid teleportation to allow the user to touch virtual objects that are considered non movable. On the other hand, teleportation of the object or hybrid teleportation may be more suitable for the user to interact with small objects or tools to perform a task at a specific location in the virtual environment. Each of these techniques also has its advantages and disadvantages. For example, teleportation of the user and teleportation of the object require additional strategies to prevent the user or the object from being teleported inside or behind other virtual objects. Hybrid teleportation solves this problem by enabling the user to manage the teleportation location themself. Nevertheless, this technique might be time-consuming and cognitive-challenging to use.

For future work, we will conduct user studies using virtual objects of different shapes, sizes, and potential mobility in various virtual scenarios to evaluate these techniques. It will also be interesting to see if experiencing tangible interactions affects the user's sense of presence and changes their behavior (e.g., with regard to obstacle avoidance) when interacting with purely virtual objects. Finally, security issues should also be considered in such an application, especially in scenarios where the tangible interaction phase alternates with the free navigation phase without tangibility support. This is because tangible props in the real world can also be considered as obstacles for the user during the free navigation.

\section{REFERENCES}

[1] B. Araujo, R. Jota, V. Perumal, J. X. Yao, K. Singh, and D. Wigdor. Snake charmer: Physically enabling virtual objects. In Proceedings of the TEI'16: Tenth International Conference on Tangible, Embedded, and Embodied Interaction, pp. 218-226, 2016.

[2] M. Azmandian, T. Grechkin, M. T. Bolas, and E. A. Suma. Physical space requirements for redirected walking: How size and shape affect performance. In ICAT-EGVE, pp. 93-100, 2015.

[3] M. Azmandian, M. Hancock, H. Benko, E. Ofek, and A. D. Wilson. Haptic retargeting: Dynamic repurposing of passive haptics for enhanced virtual reality experiences. In Proceedings of the $2016 \mathrm{CHI}$ conference on Human Factors in Computing Systems, pp. 1968-1979, 2016.

[4] D. A. Bowman, D. Koller, and L. F. Hodges. Travel in immersive virtual environments: An evaluation of viewpoint motion control techniques. In Proceedings of IEEE 1997 Annual International Symposium on Virtual Reality, pp. 45-52. IEEE, 1997.

[5] A. S. Carlin, H. G. Hoffman, and S. Weghorst. Virtual reality and tactile augmentation in the treatment of spider phobia: a case report. Behaviour research and therapy, 35(2):153-158, 1997.

[6] W. Chen, A. Plancoulaine, N. Férey, D. Touraine, J. Nelson, and P. Bourdot. $6 \mathrm{DoF}$ navigation in virtual worlds: comparison of joystickbased and head-controlled paradigms. In Proceedings of the 19th ACM Symposium on Virtual Reality Software and Technology, pp. 111-114, 2013. 
[7] L.-P. Cheng, L. Chang, S. Marwecki, and P. Baudisch. iturk: Turning passive haptics into active haptics by making users reconfigure props in virtual reality. In Proceedings of the 2018 CHI Conference on Human Factors in Computing Systems, p. 89. ACM, 2018.

[8] L.-P. Cheng, T. Roumen, H. Rantzsch, S. Köhler, P. Schmidt, R. Kovacs, J. Jasper, J. Kemper, and P. Baudisch. Turkdeck: Physical virtual reality based on people. In Proceedings of the 28th Annual ACM Symposium on User Interface Software \& Technology, pp. 417-426, 2015.

[9] J. J. Gibson. The theory of affordances. In J. B. Robert E Shaw, ed., Perceiving, acting, and knowing: toward an ecological psychology, pp. pp.67-82. Hillsdale, N.J. : Lawrence Erlbaum Associates, 1977.

[10] M. J. Habgood, D. Moore, D. Wilson, and S. Alapont. Rapid, continuous movement between nodes as an accessible virtual reality locomotion technique. In 2018 IEEE Conference on Virtual Reality and 3D User Interfaces (VR), pp. 371-378. IEEE, 2018.

[11] H. G. Hoffman. Physically touching virtual objects using tactile augmentation enhances the realism of virtual environments. In Proceedings. IEEE 1998 Virtual Reality Annual International Symposium (Cat. No. 98CB36180), pp. 59-63. IEEE, 1998.

[12] B. E. Insko, M. Meehan, M. Whitton, and F. Brooks. Passive haptics significantly enhances virtual environments. $\mathrm{PhD}$ thesis, University of North Carolina at Chapel Hill, 2001.

[13] L. E. Kavraki, P. Vestka, J. claude Latombe, and M. H. Overmars. Probabilistic roadmaps for path planning in high-dimensional configuration spaces. IEEE Transactions on Robotics and Automation, 12:566-580, 1996.

[14] L. Kohli, E. Burns, D. Miller, and H. Fuchs. Combining passive haptics with redirected walking. In Proceedings of the 2005 international conference on Augmented tele-existence, pp. 253-254, 2005.

[15] L. Kohli, M. C. Whitton, and F. P. Brooks. Redirected touching: The effect of warping space on task performance. In 2012 IEEE Symposium on 3D User Interfaces (3DUI), pp. 105-112. IEEE, 2012.

[16] L. Kohli, M. C. Whitton, and F. P. Brooks. Redirected touching: Training and adaptation in warped virtual spaces. In 2013 IEEE Symposium on 3D User Interfaces (3DUI), pp. 79-86. IEEE, 2013.

[17] A. Kotranza and B. Lok. Virtual human + tangible interface $=$ mixed reality human an initial exploration with a virtual breast exam patient. In 2008 IEEE Virtual Reality Conference, pp. 99-106. IEEE, 2008.

[18] J. Lacoche, N. Pallamin, T. Boggini, and J. Royan. Collaborators awareness for user cohabitation in co-located collaborative virtual environments. In Proceedings of the 23rd ACM Symposium on Virtual Reality Software and Technology, p. 15. ACM, 2017.

[19] W. A. McNeely. Robotic graphics: a new approach to force feedback for virtual reality. In Proceedings of IEEE Virtual Reality Annual International Symposium, pp. 336-341. IEEE, 1993.

[20] A. L. Simeone, E. Velloso, and H. Gellersen. Substitutional reality: Using the physical environment to design virtual reality experiences. In Proceedings of the 33rd Annual ACM Conference on Human Factors in Computing Systems, pp. 3307-3316. ACM, 2015.

[21] E. A. Suma, Z. Lipps, S. Finkelstein, D. M. Krum, and M. Bolas. Impossible spaces: Maximizing natural walking in virtual environments with self-overlapping architecture. IEEE Transactions on Visualization and Computer Graphics, 18(4):555-564, 2012.

[22] T. Wei $\beta$ ker, A. Kunert, B. Frohlich, and A. Kulik. Spatial updating and simulator sickness during steering and jumping in immersive virtual environments. In 2018 IEEE Conference on Virtual Reality and 3D User Interfaces (VR), pp. 97-104. IEEE, 2018.

[23] T. Weissker, A. Kulik, and B. Froehlich. Multi-ray jumping: comprehensible group navigation for collocated users in immersive virtual reality. In 2019 IEEE Conference on Virtual Reality and 3D User Interfaces (VR), pp. 136-144. IEEE, 2019. 